\title{
LA FORTUNA DEL DE CORPORE HOBBESIANO EN LA HISTORIA DE LA MECÁNICA: SU RECEPCIÓN EN LA OBRA DE G.W. LEIBNIZ (670)
}

\author{
Alberto Guillermo RANEA \\ Universidad Torcuato Di Tella. Buenos Aires (Argentina)
}

Resumen: Quisiera sugerir brevemente en este trabajo que el estudio comparativo de las formas de exposición de concepciones filosoficas antagonistas en el siglo XVII puede resultar de importancia para la investigación de su contenido. Para ello me concentraré en el caso de la posible existencia de una semejanza estructural entre el De corpore, de Thomas Hobbes, $y$ dos textos de G. W. Leibniz, Theoria motus abstracti e Hypothesis physica nova, de 1670, en los que presenta su primera teoria del movimiento o phoronomia.

La posibilidad de que la estructura de textos de diferentes autores sirva como pista para comprender mejor su contenido me fue sugerida por la lectura de The Politics of Motion. The World of Thomas Hobbes, de Thomas Spragens ${ }^{1}$. Este libro recibió críticas severas entre historiadores y filósofos pero su acogida fue calurosamente favorable entre sociólogos de la ciencia. El secreto de su éxito entre representantes de los social studies of science, como Steven Shapin, radicaba en la tesis central de Spragens —derivada de las interpretaciones de Watkins y Macpherson 2 - según la cual existe una interacción entre la filosofia natural y la filosofia política en Hobbes. Esta interacción habría sido de tal magnitud como para que Spragens transformara la cautelosa afirmación de la "considerablen interacción recíproca en la franca declaración de que el contenido final de

1 The University of Kentucky Press, Lexington, 1973.

2 J.W.N. WATKINS, Hobbes System of Ideas, Hutchinson, 1965; Macpherson, "Introduction» to Th. Hobbes, Leviathan, London, 1965. 
la filosofia política de Hobbes habría recibido el impacto de su filosofia natural, pero no a la inversa como cabría esperar si se tratara de una genuina interacción.

La tesis de Spragens se completaba con la aparentemente inocua y trillada afirmación de que Hobbes habría accedido a su tan impactante filosofía natural gracias a que el conocimiento de la mecánica de Galileo Galilei le habría reabierto las puertas de la naturaleza ${ }^{3}$. El apoyo de las teorías galileanas habría sido decisivo para que Hobbes pudiera combatir a su principal antagonista en filosofia, Aristóteles ${ }^{4}$. De acuerdo con Spragens, esto explicaría por qué algunos de los más influyentes intérpretes del pensamiento de Hobbes -entre ellos Leo Straussseñalan la presencia de Aristóteles en Hobbes a pesar del antagonismo con la tradición en la que se habia formado, einfused with Aristotelian premisses" ${ }^{5}$. La aparente paradoja se develaría cuando señala Spragens que las ideas de Aristóteles han configurado las de Hobbes en el sentido de que la obra de Hobbes ais ... heavily shaped by, indeed saturated by, the very Aristotelian framework whose content he (= Hobbes) is rejecting” ${ }^{6}$. Así, por ejemplo, Hobbes habria estructurado sus reflexiones antropológicas sobre las reflexiones correspondientes de Retorica ${ }^{7}$. De manera más explícita aún, Spragens afirma que Hobbes aplica de modo sistemático su modelo básico de movimiento a todos los constituyentes del universo de la misma manera que Aristóteles aplicara el suyo también a toda la realidad. Más aún, la aplicación universal de un paradigma radical ("root-paradigm" es la expresión de Spragens), es un paso prefigurado por la cosmología aristotélica. La diferencia crucial entre Aristóteles y Hobbes estría en la naturaleza del modelo explícito que usan, pero "[t] the formal patterns of Hobbes's cosmology are ... taken as prefabricated from the structure of the Aristotelian framework" ${ }^{8}$.

Aunque no creo acertado seguir a Spragens en su omnívora búsqueda de semejanzas estructurales y antagonismos de contenido entre Hobbes y Aristóteles, creo que su estrategia general puede servir para revelar el plan leibniciano al redactar la Hypothesis physica nova. En efecto, un análisis detallado del De corpore, de Hobbes, y del texto leibniciano recién mencionado nos revela que ambos tienen

Thomas A. SPRAgens, Jr. The politics of Motion, 1973, 36.

4 He abordado este problema en mi trabajo inédito "Leibniz lecteur de Hobbes, lecteur de Galilée?», leido en el coloquio "Leibniz lecteur de Hobbes», Paris, 27-29 de junio de 1996.

5 Thomas A. SPRAGENS, Jr. The politics of Motion, 1973, 38.

6 Thomas A. SPRAGENS, Jr. The politics of Motion, 1973, 38-9.

7 Thomas A. SPRAGENS, Jr. The politics of Motion, 1973, 39.

8 Thomas A. SPRAGENS, Jr. The politics of Motion, 1973, 68-69. 
una estructura atractivamente semejante. Como en el caso del antagonismo entre Hobbes y Aristóteles, también tendríamos en Leibniz un pensamiento configurado de acuerdo con la estructura del pensamiento antagonista. Esto significa, en una aproximación primera, que ambos presentan el mismo orden de exposición de los temas, algo que con paciencia y tiempo suficiente no es muy difícil de advertir. Pero sólo con ello no habremos ganado mucho en la comprensión del verdadero problema; la estructura del texto no es interesante por sí misma, sino por lo que devela y a la vez oculta. Ella puede orientarnos hacia el motivo que llevara a Leibniz a no estar satisfecho con la solución de Hobbes al problema de la fundamentación de la mecánica. De allí habría nacido la phoronomia de Leibniz.

"Phoronomia", "Phorologia", "Ars tornatoria, sive Motuum vestigiis» son algunos de los nombres de una ciencia del movimiento puramente geométrica que Leibniz concibió entre mediados de 1669 y comienzos de 1670 con el propósito de presentarse ante la Royal Society of London. El contexto de este proyecto lo delinean las propuestas de Christiaan Huygens, Christopher Wren y John Wallis para corregir las leyes cartesianas del movimiento. El resultado de este temprano interés de Leibniz por el movimiento y los fenómenos de choque es la Hypothesis physica nova, una obra publicada en Mainz y en Londres por el editor de la Royal Society, J. Martyn. El texto fue impreso en dos partes, en primer lugar la «Theoria motus abstracti seu rationes motuum universales, a sensu et Phaenomenis independentes", dedicada a la Académie Royale des Sciences de Paris, y la segunda, la "Theoria motus concreti seu Hypothesis de rationibus phaenomenorum nostri Orbis", conocida como la Hypothesis physica nova, dedicada a la Royal Society of London. De acuerdo con la autorizada información de Martial Gueroult, el objetivo que Leibniz persegúa con estos textos era el de superar las limitaciones de la ciencia contemporánea del movimiento tal como la habían desarrollado Galileo, Huygens y Wren. Siempre siguiendo a Gueroult, Leibniz se propuso reemplazar el método empírico que aquéllos habrian seguido al derivar las leyes del movimiento de observaciones puras o de la combinación de observación y racionamiento, por un procedimiento puramente racional «qui deduira les movements sensibles de principes abstraits et purement rationnels et opéréra, comme la géométrie, par construction a priori» ${ }^{9}$.

La influencia decisiva de Thomas Hobbes sobre la filosofia natural de Leibniz ha sido repetidas veces subrayada desde la aparición del artículo de Arthur

9 Martlal Gueroult, Leibniz. Dynamique et Métaphysique. Paris, Aubier, 1967, 8. 
Hannequin titulado "La première philosophie de Leibniz", incluído en la edición de 1908 de sus Etudes d'histoire des sciences et d'histoire de la philosophie ${ }^{10}$. Aunque los intérpretes hayan luego concentrado su atención en la influencia sobre el joven Leibniz de otros personajes, en particular Pierre Gassendi, Martin Weigel o J. Thomasius, el interés juvenil de Leibniz por la filosofía natural de Hobbes es indiscutible e insoslayable. Entre los especialistas que en las últimas dos décadas han señalado la deuda de Leibniz con Hobbes quisiera destacar a Catherine Wilson. Ella afirma acertadamente que la distinción entre "abstracto" y "concreto" en los títulos de las dos partes de Hypothesis physica nova deriva de la implícita diferenciación entre philosophia prima y physica tal como aparece en Leviathan ${ }^{11}$. La pista de la conexión hobbesiana es, a mi juicio, muy sugestiva, así como son inobjetables los comentarios de Wilson acerca de la supuesta rareza de la división

uf the theory of motion into an abstract component and a concret component .... ${ }^{12}$

Creo sin embargo que podríamos tener una mejor comprensión del significado y alcance de la división entre un plano abstracto y otro concreto si en lugar de recurrir al Leviathan prestáramos atención al más desatendido, al menos en lengua española, De corpore ${ }^{13}$. Si lo hiciéramos descubriríamos que las dos partes del texto leibniciano, aparentemente desconectadas entre sí, son dos partes del mismo proyecto, un proyecto que es paralelo al que Hobbes presenta en el De corpore. En otras palabras, Theoria motus abstracto y Theoria motus concreti constituirían juntas la versión leibniciana del De corpore de Hobbes. La estructura es la misma, el objetivo del proyecto también. En ambos casos se trata de fundamentar la nueva ciencia del movimiento en términos de una philosophia prima, pero hay algo en el texto de Hobbes que mueve a la crítica de Leibniz. Dicho con mayor precisión, la phoronomia de Leibniz, tal como aparece en esas dos obras sin conexión aparente entre sí, sería

10 Paris, Alcan, 1908.

11 Catherine WILSON, Leibniz' Metaphysics. A Historical and Comparative Study. Manchester, Manchester University Press, 1989, 52.

12 Ib., 51.

13 Una generosa excepción es la edición espafiola, Thomas HOBBES, Tratado sobre el cuerpo. Introducción, traducción y notas de Joaquín RoDRiguez FEO. Madrid, Editorial Trotta, 2000 . 
la primera expresión del desagrado que Leibniz experimenta ante el modo en que Hobbes trata de fundamentar la nueva ciencia del movimiento.

No es difícil documentar el conocimiento precoz que Leibniz tuvo del del De corpore. Junto al De cive, es el texto hobbesiano citado con mayor frecuencia en los papeles leibnicianos anteriores a 1672. Curiosamente, a pesar de los frecuentes comentarios adversos a su autor, Leibniz no rechaza las ideas del $D e$ corpore. Más aun, en numerosas oportunidades se apoya en él como autoridad en filosofía natural. En Notae Grammaticae ${ }^{14}$, Leibniz menciona al De corpore entre las obras cuyas definiciones podrían ser usadas para su proyecto de physica. Debemos prestar atención a este ejemplo. Leibniz había ya acusado en varias oportunidades a Hobbes por haber hecho de la verdad un capricho del arbitrio humano: si la verdad se demuestra en una cadena de definiciones y éstas son arbitrarias y nominales, es fácil concluir la arbitrariedad de toda verdad. Leibniz opone a esta concepción la necesidad de recurrir a definiciones reales que contengan la posibiliad de lo definido, es decir, definiciones causales que indiquen la manera en que la cosa definida pueda ser producida. Hasta lo que yo he podido advertir, y a pesar de la importancia de este punto para la distancia que Leibniz quería tomar del hobbismo, el De corpore nunca es mencionado como ilustración del abuso de definiciones arbitrarias. Incluso si los parágrafos 8 y 9 del capítulo 3 del De corpore que contienen el enunciado de la doctrina hobbesiana de las definiciones ${ }^{15}$ merecen fuertes críticas en la carta de Leibniz a Jean Gallois de $1672^{16}$, Leibniz emplea sin revisión la mayoría de las innumerables definiciones que da Hobbes en el De corpore. A modo de ejemplo citaré las definiciones de "cuerpo fluído" y de "cuerpo duro" en la carta a Henry Oldenburg del 28 de septiembre de $1670{ }^{17}$, el uso de las definiciones de "mayor" y de "menor" en Georgius Ulicovius Lithuanus ${ }^{18}$, las de experientia y de prudentia en Notae ad Jacobum Thomasium ${ }^{19}$, y la de "memoria» en el De Reminiscencia et reflexione ${ }^{20}$. Podemos agregar a esto que Leibniz tampoco rechaza las explicaciones que Hobbes construye sobre tales definiciones.

14 G. W. LEIBNIZ, A VI.4.A, 108.

15 Thomas HOBBES, De corpore, Opera philosophica quae latini scripsit ..., London, Vol. 1,1839 [en adelante, "OL"], I, 32-33.

16 A III.1, 13 y 16.

17 A II.1, 63-64.

18 A IV.1, 49.

19 A VI.1, 60.

20 A VI.3, 517. 
Para sólo citar algunos casos entre muchos, mencionaré la explicación del movimiento de la Tierra en el ecuador y su influencia sobre la acción de la grave$\mathrm{dad}^{21}$, la del movimiento del sol alrededor del centro del universo ${ }^{22}, \mathrm{y}$ también la explicación de fuentes y pozos surgentes en las montañas ${ }^{23}$.

Todas estas definiciones que Leibniz acepta sin modificación provienen de la Segunda y de la Tercera Parte del De corpore. Las explicaciones de numerosos fenómenos construídas sobre esas definiciones pertenecen a su Cuarta Parte. En ninguna de ellas encuentra Leibniz motivos para rechazarlas como ejemplos del repudiable "hipernominalismo" de Hobbes ${ }^{24}$. ¿Cuál es el motivo de su insatisfacción con el De corpore y de la consecuente necesidad de reformarlo? ¿Por qué sostiene el joven Leibniz en el De ratione perficiendi et emendandi encyclopaediam Alstedii, que se debería incluir al De corpore en el proyecto de la enciclopedia pero con correcciones? ${ }^{25}$ Creo que la clave está en la expectativa que Hobbes crea en el joven de Leipzig cuando aquél enuncia en el De corpore que una definición es ha de mostrar la ucausa probable de la generación de la cosam. En otros términos, el problema estaría en la manera inadecuada en la que Hobbes intenta producir el pasaje de la philosophia prima a la physicam, sive causas rerum in mundo, es decir del plano metafisico-abstracto al físico-concreto. Creo que el eje en torno al cual gira la argumentación hobbesiana es la definición que da del conatus en términos de movimiento imperceptible:

«Primo, definiemus conatum esse motum per spatium et tempus minus quam quod datur, id est, determinatur, sive expositione vel numero assignatur, id est, per punctumn" ${ }^{26}$.

21 Propositiones quaedam physicae. Dritter Entwurf, A VI.3, 24.

22 Hypothesis de systemate mundi, A VI.2, 298.

23 Hypothesis physica nova, A VI.2, 235.

24 «Ex hac ima regula Nominales deduxerunt, omnia in rerum natura explicari posse, etsi universalibus et formalitatibus realibus prorsus careatur, qua sententia nihil verius, nihil nostri temporis philosopho dignius, usque adeo, tu credam ipsum Ockamum non fuisse Nominaliorem, quam nunc est Thomas HOBBES, qui, tu, verum fatear, mihi plusquam Nominalis videtur. Marii Nizolii de veris principiis et vera ratione philosophandi libri IV, A VI, 2,428 .

25 «Encyclopaediae igitur necessaria sunt primo elementa verae philosophiae accurate demonstrata. Huc inserendus Thomas HOBBES de Corpore et de Cive integer, passim tamen emendatus.n A VI. 2, 395.

26 Thomas HOBBES, OL, I, 177. 
Esta definición pertenece, a mi juicio, al plano metafísico-abstracto y en tal calidad Leibniz la acepta a pesar de las reservas que hace a la noción de punto que presupone. Las dudas y objeciones de Leibniz surgen ante la manera en que Hobbes utiliza al conatus como concepto central de la descripción de las causas posibles de la generación de los fenómenos. En su función de nexo en la transición de lo abstracto a lo concreto, el conatus hobbesiano adquiere propiedades estáticas que se confunden con las asignadas en abstracto por la definición nominal que acabamos de citar. Por el contrario, Leibniz considera necesario distinguir la dimensión metafísico-abstracta del conatus de cualquiera de sus posibles interpretaciones en términos de fenómenos físicos como lo son los estáticos. Esta diferencia se ve con claridad cuando, al referirse al $D e$ corpore en sus Notae ad Danielem Stalium, Leibniz afirma que si bien la philosophia prima surge de las definiciones dadas por Hobbes, la física lo hace de los fundamentos ofrecidos por los sentidos ${ }^{27}$. El problema es que Hobbes no ha logrado relacionar los dos planos, es decir, no ha logrado dar una explicación satisfactoria del pasaje de las definiciones nominales a las definiciones reales.

Creo, sin embargo, que Leibniz encontró en el De corpore una solución posible al problema que planteaban las definiciones nominales. Un breve texto leibniciano de 1676 - basado, de acuerdo con sus editores, en el capítulo I, parágrafos 4 y 5 , del De corpore- afirma:

"Proprietas omnis quae continet efficientem rei causam, seu generationem, sufficit ad omnia eius atribute invenienda. Scilicet quicquid generationem rei continet, ut loquitur Hobbes» ${ }^{28}$.

Pero "causas o generaciones» es la expresión con la cual Hobbes caracteriza en el capítulo 1, parágrafo 2 del De corpore al objeto de la explicación filosófi$\mathrm{ca}^{29}$. Leibniz habría comprendido que Hobbes no se ha limitado a formular definiciones arbitrarias de los términos explicativos utilizados en el De corpore. Leibniz habría además podido aprender en este texto que la condición de que las definiciones sean nominales solamente tiene sentido dentro de los límites

27 "Et Thomas Hobbius qui suum opus de corpore in duas partes, in Philosophiam primam, s. abstractam ab existentia; et Physicam s. Causas rerum in Mundo stantium divisitm. Notae ad Danielem Stahlium (Compendium Metaphysicum), A VI. 1, 22.

28 «Aufzeichnungen», A VI. 3, 388.

29 Thomas HOBBES, OL, I, 2. 
de la philosophia prima sive abstractam ab existentia. En el reino de lo concreto es necesario completar esas definiciones por medio de una explicación genético-causal de su objeto.

Creo que esta interpretación nos permite conjeturar por qué motivo Leibniz sostiene en el De ratione perficiendi et emendandi encyclopaediam Alstedii que se debería incluir el De corpore en la enciclopedia pero una vez efectuadas algunas correcciones ${ }^{30}$. Dadas las dudas leibnicianas acerca del hipernominalismo de Hobbes, todo indicaría que las correcciones deben practicarse en las definiciones nominales del De corpore. Sin embargo, creo que Leibniz piensa que es necesario corregir la manera en que Hobbes explica la derivación de la física a partir de la philosophia prima, es decir el pasaje de la philosophia prima sive abstractam ab existentia a la physica. En juego estaría la cuestión de la capacidad de una teoría abstracta del movimiento para explicar los fenómenos físicos concretos. El caso más acuciante en la época era el de la teoría galileana del movimiento, a la que René Descartes y el mismo Leibniz criticaban por descuidar el papel del medio en la determinación de las leyes del movimiento. En este contexto quisiera sugerir que la estructura del programa que Hobbes desarrolla en el De corpore ha determinado los pasos que Leibniz siguiera en la elaboración de su Hypothesis physica nova. Me concentraré para mostrarlo en el término sobre el cual gira la transición de lo abstracto a lo concreto en la obra de Hobbes: la noción de conatus. No pretendo ofrecer una interpretación original del término, sino indicar simplemente en qué ocasiones aparece y desaparece el conatus en el De corpore y en Hypothesis physica nova.

Como se sabe, conatus es definido en el capítulo 15 del De corpore, es decir, en el primer capitulo de la Tercera parte, titulada De rationibus motuum, et magnitudinum. Sin embargo, conatus desaparece del texto hasta el momento en que en un Corolario del capítulo 21 se lo utiliza para describir el caso de una esfera que gira con un movimiento simple en un medio fluido homogéneo ${ }^{31}$. A partir de ese momento hasta el capítulo 24 que cierra la tercera parte, conatus es puesto en relación con diferentes situaciones propias de la estática, en particular de la

30 «Encyclopaediae igitur necessaria sunt primo elementa verae philosophiae accurate demonstrata. Huc inserendus Thomas HOBBES de Corpore et de Cive integer, passim tamen emendatus." A VI. 2, 395.

31 «8. Si in medio liquido moto ut dictum est circulariter, natare intelligatur aliud mobile sphaericum non liquidum partes liquidi in ipsum impingentes conatus habebunt sese undequaque, per superficiem ipsius corporis non liquidi, expandendi", Thomas HOBBES, OL, 1, 267. 
hidrostática. En esos cuatro capírulos, conatus pierde poco a poco el carácter cinemático que tenía en su definición abstracta y adquiere una dimensión concreta que le vuelve indispensable para las explicaciones fisicas hipotéticas de la Cuarta parte del De corpore. Si se me permite el anacronismo, querría sugerir la analogía entre la estructura del De corpore con lo que se conoció en la filosofía de la ciencia del siglo XX como un «sistema formal más interpretación". Siguiendo esta comparación, la Tercera Parte del De corpore, hasta el capítulo 20 inclusive, sería el equivalente a un cálculo formal: Hobbes presenta allí las combinaciones posibles de los términos definidos nominalmente y que carecen de un referente empírico directo; la Cuarta Parte, a partir del capítulo 25, presentaría los equivalentes a modelos o interpretaciones relacionados con los fenómenos que se quiere explicar con la teoría desarrollada en abstracto. Finalmente, y para completar la analogía, los capítulos 21 a 24 funcionarían como reglas informales de correspondencia que pondrían en relación términos definidos nominalmente, como conatus, con un número muy variado de diferentes fenómenos.

Creo que las dudas de Leibniz acerca del programa hobbesiano aparecen precisamente alrededor de estas "reglas informales de correspondencia" entre la definición abstracta de conatus y los fenómenos físicos a explicar. El término conatus queda así cargado de claras y explícitas connotaciones estáticas que se confunden con su definición nominal abstracta y que no han dejado de sorprender y escandalizar a los comentaristas. En efecto, entre los ejemplos con los que Hobbes intenta hacer plausible la definición de conatus como término clave de su física, encontramos que una bala de plomo descendería con mayor conatus que una de lana ${ }^{32}$. Este texto ha golpeado profundamente a Frithiof Brandt, autor de la hasta ahora única exposición exhaustiva de la filosofia y ciencia natural de Hobbes. Brandt confesó que carecía del coraje moral necesario para admitir que Hobbes había sostenido una afirmación tan antigalileana y tan alejada de la concepción clásica de la aceleración constante producida por la gravedad ${ }^{33}$. El problema que enfrentaba Hobbes sin embargo le obligaba a esta decisión. Si, por un lado, el conatus estaba llamado a servir de fun-

32 «Eodem modo si sint duo motus simul incipientes et simul desinentes, conatus eorum erunt aequales vel inaequales in ratione velocitatum; quemadmodum videmos majore conatu descendere pilam plumbeaum, quam laneam". Thomas HOBBES, OL, I, 178.

33 "But we honestly confess that we do not possess moral courage enough to attribute to HOBBES, the professed devotee and admirer of Galileo, a line of thought which presupposes that in an essential particular he had not understood the master [...] Just as matter-of course as it 
damento de las mediciones de los fenómenos físicos, era dificultoso atribuirle un valor numérico determinado, puesto que el conatus es definido como un movimiento por una distancia y en un tiempo que se ha decidido ignorar, no tomar en cuenta. Hobbes considera, sin embargo, que es posible atribuirle valores comparativamente a diferentes conatus, es decir comparándolos en una escala de más y de menos. A pesar de la escrupulosa incredulidad de Brandt, Hobbes afirma que cuerpos de diferentes tipos de materia caerán con velocidades diferentes, lo que permitiría establecer un valor proporcional relativo para sus conatus. De esta manera se podría evaluarlos comparando las diferentes distancias que cada uno de ellos recorre en un tiempo dado. La única condición que deben cumplir es la de comenzar y terminar simultáneamente.

Si el tiempo es el phantasm del movimiento uniforme ${ }^{34}$, y éste a su vez es definido como la coincidencia del comienzo y de la finalización de movimientos diferentes ${ }^{35}$, los distintos conatus serán evaluables en función de sus velocidades diferentes en tiempos iguales, es decir, como movimientos uniformes. Así, los diferentes conatus de la bala de plomo y la de lana indican que ellas caerán con velocidades uniformes diferentes. A pesar del desacuerdo de Brandt, Hobbes establece así una clara relación entre el conatus y las velocidades uniformes de los desplazamientos virtuales de la estática; más aún, Hobbes nunca relaciona en el De corpore al conatus con la aceleración de la caída libre, para cuyo análisis reserva el término impetus. En otras palabras, según los supuestos del De corpore, conatus, aunque estrechamente ligado con el impetus, expresa una dimensión de la acción de los cuerpos que difícilmente podría relacionarse con la dimensión de la aceleración. En otros términos, el conatus serviría para explicar las "causas o generaciones» de los fenómenos estáticos; por su parte, el impetus sería el elemento constitutivo de la generación de los fenómenos de la aceleración. Esto explicaría por qué en el De corpore Hobbes no ha utilizado nunca al conatus como elemento infinitesimal de la aceleración.

seems to the popular consciousness to believe that a bullet of lead, falling simultaneously with and from the same height as a ball of wool, will reach the ground before the ball of wool, just as impossible is it to the person who has merely once read the Discorsi with understanding to believe anything but that they reach the ground at exactly the same timew, Frithiof Brandt, Thomas Hobbes Mechanical Conception of Nature, Copenhagen, London, 1928, 298.

34 "Tempus enim quod est phantasma motus non nisi per expositum motum aestimatur", Th. HOBBes, OL, I, 101.

35 "Atque ea velocitas, qua aequalibus temporis partibus aequales transeuntur longitudines, velocitas sive motus uniformis dicitur, Th. HOBBES, OL, I, 101. 
La frecuente asociación en los escritos juveniles de Leibniz del conatus con fenómenos hidrostáticos sugiere una lectura atenta y temprana del texto hobesiano. Se trataría de una lectura que busca dar una mayor precisión al proceso por el cual Hobbes transforma la dimensión cinemática del conatus en propiedades estáticas. Aun a riesgo de parecer excesivamente audaz, me atrevería a sugerir que la Theoria motus abstracti podría ser equivalente a los capítulos 15 a 20 de la tercera parte del De corpore, y la Theoria motus concreti como la versión leibniciana de la Cuarta parte del texto hobbesiano. Como para Hobbes, también para Leibniz el problema a resolver es la transición de lo abstracto a lo concreto. En el caso de los textos leibnicianos, dicha transición estaría esbozada en el Problema undécimo de la Theoria motus abstracti:

"et generatim in AETHERE, per Hypotheses nostras, cujus cum motu potius quam suo corpora sensibilia ferantur, habebunt ab hoc subtili portitore [...] motum circularem simplicem Hobbianum .... ${ }^{36}$

Si recordamos que Hobbes apela al movimiento circular simple para iniciar el proceso de interpretación concreta del término abstracto conatus, este texto, en el que Leibniz señala la conveniencia de explicar la generación de dicho movimiento en términos de la "sutilidad del éter", señalaría la insuficiencia de la manera en que Hobbes propone el pasaje de lo abstracto a lo concreto. En palabras de Leibniz que parecen confirmar mi suposición,

"[p]otest ergo assumto solo Aethere Theoria motus concreti derivari ex theoria motus abstracti, et solvi hoc problema generale: Omnes motus sensibiles explicaren" ${ }^{37}$.

A partir del "Problema general», encontramos, a mi juicio, en la Theoria motus abstracti una crítica a la manera en que Hobbes plantea el proceso de concretización que el término conatus sufre en los capítulos 21 a 24 del De corpore. En este contexto la sinonimia de conatus y pressio, frecuente en los textos de Leibniz redactados durante su estancia parisina, no representaría una diferente definición de conatus sino un nuevo ensayo de interpretación concreta del término definido en abstracto.

36 G. W. LeIBNIZ, Theoria motus abstracti, A VI.2, 271.

37 Ibid. 